\title{
New determination of the mass of Pallas
}

\author{
E. Goffin
}

\author{
Aartselaarstraat 14, 2660 Hoboken, Belgium \\ e-mail: Goffin@twi.agfa.be
}

Received 13 September 2000 / Accepted 6 October 2000

\begin{abstract}
Until very recently, the mass of minor planet 2 Pallas had been determined from its gravitational effects on only 1 Ceres and the planet Mars. An independent confirmation was therefore highly desirable. This paper presents individual mass determinations based on close encounters with 16 other minor planets, as well as a simultaneous solution using all objects. The resulting value for the mass of Pallas, $(1.17 \pm 0.03) 10^{-10} M_{\odot}$, essentially confirms the result from Ceres alone.
\end{abstract}

Key words. astrometry - minor planets, asteroids - planets and satellites: individual: 2 Pallas

\section{Introduction}

The first determination ever of the mass of Pallas was made by Schubart (1974). He revised it in Schubart (1975) and later gave a better value (Schubart \& Matson 1979) using an improved value for the mass of Vesta. His work was based on the near 1:1 mean-motion resonance between the orbits of Ceres and Pallas. This resulted in a series of moderately close approaches in the first part of the 19th century, leaving observable gravitational effects in the co-ordinates of both minor planets. Since then, the pair is widening and the minimum distances are increasing steadily. Hence, the accuracy of the mass determination largely depends on the oldest - and least accurate observations.

A different technique was used by Standish \& Hellings (1989). Exploiting the high accuracy of the Mars data from the Viking Landers, they determined the masses of Ceres, Pallas and Vesta. Later, different values were obtained from the solution of the JPL ephemerides DE403 (Standish et al. 1995) and DE405 (Standish 1998). Hilton (1999) simultaneously determined the masses of Ceres, Pallas and Vesta as part of his work on the ephemerides of 15 of the largest asteroids. He obtained a substantially larger mass for Pallas and a correspondingly lower result for Ceres.

No independent determination of the mass of Pallas based on other perturbed minor planets existed until very recently. This is not surprising in view of the fact that the orbital inclination of Pallas is $35^{\circ}$, which keeps it out of the main asteroid belt for most of the time. As the work on this paper drew to a close, a new result was published by Michalak (2000). He calculated mass values for Pallas from perturbations on 8 minor planets, but retained only the results from 582 Olympia and 9 Metis for his final result.

Here I present new mass determinations of Pallas, both individual and simultaneous, based on close encounters with several other minor planets.

\section{Minor planet selection and observations}

Possible candidates for mass determinations were selected as follows. For each of the first 10000 numbered minor planets, all close approaches to Pallas were computed from their respective discovery date until the year 2010. For each of these encounters, the minimum distance $r_{0}$ to Pallas and the corresponding relative velocity $v_{0}$ were computed. Next, the change in heliocentric velocity $\Delta v$ of the perturbed minor planet was calculated from (Carpino \& Knežević 1996):

$\Delta v=\frac{2 k^{2} m}{r_{0} v_{0}}$

where $k$ is the Gaussian gravitational constant and $m$ is the mass of the perturbing minor planet. All candidates were then ordered according to the value of $\Delta v$ and the hundred most promising cases were retained for further investigation.

Success of a mass determination depends not only on the geometry of the mutual approaches, but also on the number and distribution of the observations. Therefore, the orbit of each of the selected asteroids was tested on its sensitivity to perturbations by Pallas and in most cases an actual mass determination was tried. Those that gave meaningful results were retained for the final solution. 
Table 1. Mass determinations of Pallas. The table gives: minor planet number and name, no. of observations used, no. of oppositions, time interval covered by the observations, mass of Pallas and standard deviation, approaches to Pallas $<0.2$ AU and year. An ${ }^{(*)}$ behind the name indicates that the minor planet is near the 1:1 resonance with Pallas

\begin{tabular}{|c|c|c|c|c|c|}
\hline Minor planet & Obs. & Opp. & Period & $\begin{array}{c}\text { Mass and rms } \\
10^{-10} M_{\odot} \\
\end{array}$ & $\begin{array}{l}\text { Close approaches }<0.2 \mathrm{AU} \\
\text { min. dist. in } \mathrm{AU} \text { (and year) }\end{array}$ \\
\hline 1 Ceres * & 3104 & 128 & 1801-1999 & $1.16 \pm 0.03$ & 0.191 (1816), 0.181 (1820), 0.189 (1825), 0.195 (1829) \\
\hline 582 Olympia & 251 & 31 & 1906-1999 & $0.93 \pm 0.11$ & $0.033(1936)$ \\
\hline 27 Euterpe & 1618 & 79 & $1853-2000$ & $0.95 \pm 0.13$ & $0.180(1894), 0.061(1926)$ \\
\hline 354 Eleonora * & 1792 & 77 & $1896-2000$ & $1.31 \pm 0.16$ & $0.109(1950)$ \\
\hline 44 Nysa & 1410 & 81 & $1857-2000$ & $1.54 \pm 0.20$ & $0.117(1935), 0.095(1977)$ \\
\hline 14 Irene & 1772 & 84 & $1851-2000$ & $2.53 \pm 0.33$ & $0.056(1917)$ \\
\hline 5930 Zhiganov & 65 & 10 & $1950-2000$ & $0.90 \pm 0.33$ & $0.015(1977)$ \\
\hline 282 Clorinde & 172 & 30 & $1889-2000$ & $1.67 \pm 0.36$ & $0.028(1936)$ \\
\hline 3131 Mason-Dixon & 123 & 12 & 1954-1999 & $1.49 \pm 0.42$ & $0.012(1984)$ \\
\hline 288 Glauke $^{*}$ & 443 & 61 & 1890-1999 & $0.82 \pm 0.46$ & $0.182(1968), 0.122$ (1972), 0.103 (1977), 0.182 (1982) \\
\hline 146 Lucina & 415 & 59 & $1875-2000$ & $0.48 \pm 0.56$ & $0.072(1957), 0.194(1982)$ \\
\hline 310 Margarita * & 278 & 46 & $1891-2000$ & $1.21 \pm 0.60$ & $\begin{array}{l}0.199(1929), 0.142(1934), 0.088 \text { (1938) } \\
0.090(1943), 0.137 \text { (1947), } 0.195 \text { (1952) }\end{array}$ \\
\hline 1655 Comas Sola * & 172 & 13 & $1901-2000$ & $1.34 \pm 0.81$ & - \\
\hline 732 Tjilaki & 250 & 30 & $1912-2000$ & $2.45 \pm 0.99$ & $0.014(1977), 0.135(2000)$ \\
\hline 235 Carolina & 161 & 39 & 1883-1999 & $1.09 \pm 1.06$ & $0.055(1971)$ \\
\hline 255 Oppavia * & 166 & 37 & $1886-1999$ & $2.84 \pm 1.09$ & $0.175(1897), 0.074(1901)$ \\
\hline \multirow{3}{*}{\multicolumn{4}{|c|}{$\begin{array}{l}\text { All minor planets, weighted mean } \\
\text { All minor planets, least-squares solution } \\
\text { All minor planets except Ceres, least-squares solution }\end{array}$}} & $1.16 \pm 0.04$ & \\
\hline & & & & $1.17 \pm 0.03$ & \\
\hline & & & & $1.18 \pm 0.07$ & \\
\hline
\end{tabular}

Most of the observations were obtained from the Minor Planet Center (Cambridge, USA). They were complemented by all older, i.e. pre-1939, observations I could find in the literature, re-reduced whenever possible with the aid of modern star catalogues. This can be a rather time-consuming occupation, especially for low-numbered minor planets.

The observations of Ceres are different in that I used only meridian observations. For the period before 1830 , I took the observations Schubart (1976) reduced to the FK4, and added the observations (1814-1818) made by Bessel in Königsberg, with systematic corrections applied as described hereafter. The period 1830-1916 was covered by meridian observations from Greenwich, Cambridge and Edinburgh. I had reduced them to the FK5 some years ago in the way Schubart did, i.e. by applying systematic corrections for each opposition, derived from the annual star catalogues published in the same volume. The observational material for the next period, 1922-1959, consists of meridian observations reduced to the FK3 and published in Jackson (1968). After 1959, the observations were collected from several sources: USNO, Carlsberg Meridian Catalogues 1-10, Tokyo, Bordeaux, Hipparcos and USNO Flagstaff Station. These observations are reduced to the FK4, the FK5 or the ICRS.

The observations were used as published. During the computations, each computed position was transformed from the ICRS reference frame to the reference frame and catalogue system of the observation before calculating the residuals.
Table 2. Masses of perturbing minor planets

\begin{tabular}{cll}
\hline Minor planet & \multicolumn{2}{l}{ Mass in $10^{-10} M_{\odot}$} \\
\hline 1 Ceres & 4.76 & (Goffin, unpublished) \\
2 Pallas & 1.08 & (starting value) \\
4 Vesta & 1.38 & (Goffin, unpublished) \\
10 Hygiea & 0.47 & (Scholl et al. 1987) \\
52 Europa & 0.14 & (estimate) \\
511 Davida & 0.18 & (estimate) \\
704 Interamnia & 0.35 & (Landgraf 1992) \\
\hline
\end{tabular}

\section{Mass determinations}

The basic principles of, and method used for mass determinations were outlined in my previous paper (Goffin 1991) and need not be repeated here. The main difference here is the use of DE403 for the planetary positions. The perturbing major planets were Mercury through Neptune.

The perturbing minor planets and their masses are listed in Table 2. The masses of Ceres and Vesta are the mean of a substantial number of individual - as yet unpublished - results. The masses of Hygiea and Interamnia are from Scholl et al. (1987) and Landgraf (1992). The masses of Europa and Davida are estimates based on their IRAS diameters of 312 and $337 \mathrm{~km}$, and an assumed mean density of $1.8 \mathrm{~g} / \mathrm{cm}^{3}$.

The results of both the individual and combined mass determinations are listed in Table 1 , the individual results in order of increasing rms error. More results were found, but I decided to retain only those cases where the rms error is less than $1.110^{-10}$, this being the 
Table 3. Influence of the masses of Ceres, Vesta and Hygiea on the mass of Pallas. $\Delta m(1)$ is the change in the mass of Pallas for a difference of $1.010^{-10} M_{\odot}$ in the mass of the perturbing body. All values are in $10^{-10} M_{\odot}$; the standard deviation is 0.03 throughout

\begin{tabular}{c|ccc|c} 
Mass of Ceres & 4.60 & 4.80 & 5.00 & $\Delta m(1)$ \\
\hline Mass of Pallas & 1.18 & 1.17 & 1.16 & -0.05 \\
Mass of Vesta & 1.20 & 1.40 & 1.60 & $\Delta m(1)$ \\
\hline Mass of Pallas & 1.19 & 1.16 & 1.14 & -0.14 \\
& & & & \\
Mass of Hygiea & 0.30 & 0.50 & 0.70 & $\Delta m(1)$ \\
\hline Mass of Pallas & 1.12 & 1.18 & 1.23 & +0.28
\end{tabular}

estimated mass of Pallas itself. I omitted the result found from 40 Harmonia since its value $(0.32 \pm 0.11)$, despite the small standard deviation, differs too much from the other results and from the final mean value for some unknown reason. As expected, the individual determinations showed large correlations between Pallas' mass and the respective semi-major axes, typically in the range of $0.85-$ 0.95, 354 Eleonora with 0.31 being an exception.

From the individual results, I first calculated the weighted mean and its standard deviation. This is often used to combine individual results, see e.g. Carpino \& Knežević (1996). For the final result however, I preferred to simultaneously determine corrections to the osculating elements of all perturbed minor planets involved and to the mass of Pallas; see e.g. Bowell et al. (1994). This meant solving by the method of least squares a system of over 24 thousand linear equations in 97 unknowns. In this simultaneous solution, the largest correlations between the mass of Pallas and the semi-major axes are 0.90 for 1 Ceres, 0.52 for 27 Euterpe and 0.49 for 582 Olympia. For the 13 other asteroids they are in the range of 0.05 0.28 . In addition, I computed a simultaneous least-squares solution using all minor planets except Ceres.

Since the orbits of some of the test minor planets are also sensitive to the masses of Ceres, Vesta and Hygiea, and in view of the degeneracy between the masses of Ceres and Pallas reported by Hilton (1999), I repeated the combined least-squares solution with different values for the masses of these bodies. The results are summarized in Table 3 .

\section{Discussion}

In general, there are much less close approaches leading to meaningful mass determinations of Pallas than there are for Ceres and Vesta. This is largely due to the high inclination and eccentricity of the orbit of Pallas. As a consequence, the individual results show large variations, and some results may well be marginal. This is also in part due to the high correlations between the mass and the semi-major axis of the test asteroids.

Besides Ceres, the most interesting minor planet showing a clear signature of the perturbing influence of Pallas
Table 4. Overview of mass determinations of Pallas

\begin{tabular}{llll}
\hline $\begin{array}{c}\text { Mass and rms } \\
10^{-10} M_{\odot}\end{array}$ & $\begin{array}{l}\text { Perturbed } \\
\text { body(ies) }\end{array}$ & Author \\
\hline 1.3 & 0.4 & Ceres & Schubart (1974) \\
1.14 & 0.22 & Ceres & Schubart (1975) \\
1.08 & 0.22 & Ceres & Schubart (1979) \\
1.4 & 0.2 & Mars & Standish \& Hellings (1989) \\
1.05 & - & DE403 & Standish et al. (1995) \\
1.0 & - & DE405 & Standish (1998) \\
1.59 & 0.05 & Ceres+Vesta & Hilton (1999) \\
1.21 & 0.26 & (582)+(9) & Michalak (2000) \\
1.17 & 0.03 & 16 asteroids & This opus \\
\hline
\end{tabular}

definitely is 582 Olympia, which prominently figured at the top of the $\Delta v$ list. Some of the other cases, such as (27), (44) and (354), have low standard deviations more because of the large number of observations than because of favourable close approaches to Pallas. Nevertheless, the standard deviations of most of the individual determinations nicely encompass the combined result.

One might argue about the feasibility of including some of the individual results or contest the validity of the rather arbitrary cut-off criterion used, but a combined solution will anyhow give a much more thrustworthy result. The weigthed mean is virtually equal to the combined least-squares value, which is to be expected if there are no correlations between the individual results (Viateau \& Rapaport 1998). Both solutions are dominated by the result from Ceres, whose standard deviation is about 4 times smaller than that of the next best case. However, the mass obtained from the solution without Ceres is very close to the final result.

Inspection of Table 3 shows that the mass of Pallas is quite unaffected by variations in the masses of Ceres and Vesta. Since these are known at present with an uncertainty of about $0.0410^{-10} M_{\odot}$, the ensuing variations in the mass of Pallas would be only 0.002 and $0.00510^{-10}$ respectively. The influence of a change in the mass of 10 Hygiea is larger. This comes mainly from its effect on the orbit of Ceres, due to a moderately close approach of $0.184 \mathrm{AU}$ in 1950 between the two. The standard deviation given by Scholl et al. (1987) for their determination of the mass of Hygiea from the orbit of 829 Academia is $0.2310^{-10} M_{\odot}$. This would imply an uncertainty of 0.065 in the mass of Pallas. Simultaneously solving for both the masses of Pallas and Hygiea using the 16 minor planets yielded $(1.15 \pm 0.04)$ for Pallas and $(0.40 \pm 0.09)$ for Hygiea. The result for Hygiea is quite realistic, despite the fact that the 16 test objects were selected for determining the mass of Pallas, not that of Hygiea! More importantly, the correlation coefficient between the two masses turned out to be 0.65 .

Table 4 gives an overview of all mass determinations of Pallas. The new result agrees rather well with the values obtained by Schubart and Michalak and with those from DE403 and DE405, but differs markedly from the 
determination by Hilton. Hilton's result depends nearly exclusively on the orbit of Ceres. As he indicated himself, his masses for Ceres and Pallas are strongly interdependent, and he obtains a substantially lower mass for Ceres in conjunction with the higher value for Pallas.

The work by Michalak, though only based on two perturbed minor planets, gives a mass value close to the result obtained here. Not surprisingly, five of the minor planets from which he calculated a mass value for Pallas were also found by me, but there are large differences between his individual results and mine. I suspect that to a large extent these are due to my including a - sometimes large number of old observations.

\section{Conclusion}

The mass of 2 Pallas has been determined from its perturbing influence on 16 minor planets. The value obtained, (1.17 \pm 0.03$) 10^{-10} M_{\odot}$, though largely influenced by Ceres, is in accordance with most of the previous determinations.

Acknowledgements. All computations were carried out at the Computer Centre of Agfa-Gevaert N.V., Mortsel, Belgium. My sincere thanks go to Dr. E. Myles Standish (Jet Propulsion Laboratory, USA) who agreed to review this paper and made a number of valuable remarks.

\section{References}

Bowell, E., Muinonen, K., \& Wasserman, L. 1994, Asteroid mass determination from multiple asteroid-asteroid encounters, in Abstracts for Small bodies in the Solar System and their Interactions with the Planets, Mariehamn, Finland

Carpino, M., \& Knežević, Z. 1996, Determination of asteroid masses from mutual close approaches, in Proceedings of the 1st Italian Meeting of Planetary Science, Bormio, Italy, January $21-28$

Goffin, E. 1991, A\&A, 249, 563

Hilton, J. 1999, AJ, 117, 1077

Jackson, E. 1968, A.P.A.E. XX, Pt. I

Landgraf, W. 1992, A determination of the mass of (704) Interamnia from observations of (993) Moultona, in Proc. IAU Symp. 152, ed. S. Ferraz-Mello, Chaos, resonance and collective dynamical phenomena in the Solar System (Kluwer Academic Publishers, Dordrecht), 179

Michalak, G. 2000, A\&A, 360, 363

Scholl, H., Schmadel, L., \& Röser, S. 1987, A\&A, 179, 311

Schubart, J. 1974, A\&A, 30, 289

Schubart, J. 1975, A\&A, 39, 147

Schubart, J. 1976, A\&AS, 26, 405

Schubart, J., \& Matson, D. 1979, Masses and densities of asteroids, in Asteroids, ed. T. Gehrels (Univ. of Arizona Press, Tucson), 84

Standish, E. M., \& Hellings, R. W. 1989, Icarus, 80, 326

Standish, E. M., Newhall, X. X., Williams, J. G., \& Folkner, W. M. 1995, JPL Planetary and Lunar Ephemerides, DE403/LE403, JPL IOM 314.10-127

Standish, E. M. 1998, JPL Planetary and Lunar Ephemerides, DE405/LE405, JPL IOM 312.F-98-048

Viateau, B., \& Rapaport, M. 1998, A\&A, 334, 729 